\title{
Simulation Study of Swarm Intelligence Based on Life Evolution Behavior
}

\author{
Yanmin Liu, ${ }^{1}$ Ying Bi, ${ }^{2}$ Changling Sui, ${ }^{3}$ Yuanfeng Luo, ${ }^{1}$ Zhuanzhou Zhang, ${ }^{1}$ and Rui Liu ${ }^{1}$ \\ ${ }^{1}$ School of Mathematics and Computer Science, Zunyi Normal College, Zunyi 563002, China \\ ${ }^{2}$ College of Management, Shenzhen University, Shenzhen 518060, China \\ ${ }^{3}$ College of Life Science, Zunyi Normal College, Zunyi 563002, China
}

Correspondence should be addressed to Yanmin Liu; yanmin7813@163.com

Received 2 September 2015; Accepted 29 October 2015

Academic Editor: Felix T. S. Chan

Copyright (C) 2015 Yanmin Liu et al. This is an open access article distributed under the Creative Commons Attribution License, which permits unrestricted use, distribution, and reproduction in any medium, provided the original work is properly cited.

Swarm intelligence (SI) is a new evolutionary computation technology, and its performance efficacy is usually affected by each individual behavior in the swarm. According to the genetic and sociological theory, the life evolution behavior process is influenced by the external and internal factors, so the mechanisms of external and internal environment change must be analyzed and explored. Therefore, in this paper, we used the thought of the famous American genetic biologist Morgan, "life = DNA + environment + interaction of environment + gene," to propose the mutation and crossover operation of DNA fragments by the environmental change to improve the performance efficiency of intelligence algorithms. Additionally, PSO is a random swarm intelligence algorithm with the genetic and sociological property, so we embed the improved mutation and crossover operation to particle swarm optimization (PSO) and designed DNA-PSO algorithm to optimize single and multiobjective optimization problems. Simulation experiments in single and multiobjective optimization problems show that the proposed strategies can effectively improve the performance of swarm intelligence.

\section{Introduction}

Optimization has been an active area of research for several decades; in order to effectively solve these problems, many scholars have been looking for solution methods, for example, conjugate gradient methods, genetic algorithm (GA), differential evolution (DE) algorithm, ant colony (ACO) algorithm, and particle swarm optimization (PSO). These algorithms have achieved satisfactory effects in some areas, and when optimization problem has lots of local optima, these algorithms will have phenomenon and low efficiency. To better solve practical problem and improve its running efficiency, some new technologies and improved algorithms are proposed in the optimization fields.

Swarm intelligence is a new evolutionary computation technology, and it has attracted more and more researchers' attention. The theory research of swarm intelligence mainly has two algorithms: ACO and PSO. ACO derived from the food collection process of the ant colonies, while PSO originated in the simulation of simple flock social system. Based on the generation mechanisms of simulating life evolution behavior, PSO and ACO have been successfully applied to many fields. According to the genetic and sociological theory, the life evolution behavior is influenced by the external and internal factors, so exploring the external and internal environment change mechanisms will further grasp evolutionary behavior. Therefore, the internal environment, for example, the mutation and crossover operator of DNA fragment, usually will be the inner decision mechanism in the life evolution process, while the external environment, for example, organization and corporation which an individual belongs to, usually depends on an individual society property which will affect individual social behaviors.

In this paper, according to the thought of the famous American genetic biologist Morgan, "life = DNA + environment + interaction of environment + gene," we proposed the mutation and crossover operation of DNA fragments based on environmental change to improve performance of intelligence algorithms. Our thoughts come from life evolution behavior based on the theory of evolution and sociology, and the process of individual evolution and improvement not only is enslaved to the genetic effects (fragment of DNA), but also 
still has the external environment influence. In view of this, we adopted the mutation and crossover operation of DNA fragments to realize the inner change of the life evolution behavior and employed the mutation and crossover operation of each individual to simulate the external environment change process.

\section{Related Works}

2.1. Searching Engine Algorithm. Firstly, we introduce the reason why particle swarm optimization (PSO) is adopted as searching engine to explore the mechanism of the mutation and crossover operation of DNA fragments based on the environmental change. In this paper, we mainly discuss the system that affected the process of individual growth and improvement; then, according to gene and sociological theory, the evolution process of a society individual will be enslaved to the internal and external environment change. Furthermore, the internal environment usually is regarded as genetic effect factor, for example, mutation and crossover of DNA fragments, and the external environment usually is the macro factors that include the individual society properties, for example, the organizations or corporations which an individual belongs to. Additionally, in the current intelligent optimization algorithms, genetic algorithm (GA), differential evolution (DE) algorithm, ant colony (ACO) algorithm, PSO, and so forth have been successfully used in various fields, and particularly PSO, which is a random swarm intelligence algorithm with the characteristic of GA and ACO, showed fast influence in recent years. Here, the swarm of PSO may be analogy of the environment, and the dimension of PSO can describe the internal environment. Therefore, the proposed strategies in this paper will combine with PSO to conduct the simulation research.

In 1995, Kennedy and Eberhart [1] firstly proposed a new optimization model based on the bird flight and foraging and then designed a novel optimization algorithm based on the swarm behavior, that is, particle swarm optimization (PSO). After twenty-year development, PSO has been widely used in science and engineering fields and achieved good effects. PSO is a kind of evolutionary algorithm similar to GA, and it also starts from the random solutions through the iterative search. Additionally, PSO evaluates the quality of the solution through the fitness value, but it has more simple rules than GA. PSO has no crossover and mutation operation, and it tracks the current individual search path to find the global optima, which makes PSO have easy implementation and high precision.

Each individual in PSO is composed of $n$-dimensional variables, and it has a velocity $v_{i} \subset R^{n}$. In the process of evolution, each individual flies to optimization position by updating its velocity and position described by

$$
\begin{aligned}
\overline{v_{i}}(t)= & \overline{v_{i}}(t-1)+\varphi_{1} \operatorname{rand}\left(\overline{p_{i}}-\overline{x_{i}}(t-1)\right) \\
& +\varphi_{2} \operatorname{rand}\left(\overline{p_{g}}-\overline{x_{i}}(t-1)\right) \\
\overline{x_{i}}(t)= & \overline{v_{i}}(t)+\overline{x_{i}}(t-1)
\end{aligned}
$$

where $\overline{p_{i}}$ is the history best position of the individual $i$ (pbest for short); $\overline{p_{g}}$ is the best individual position in swarm (gbest for short); $\varphi_{1}$ and $\varphi_{2}$ are the speed constant of individual between 0 and $2 ; r_{1}$ and $r_{2}$ are uniformly distributed random vector between 0 and 1 .

Usually, good things are flawed, although PSO also has some defects in practical applications. For example, when PSO optimizes an optimization problem with many local optima, the swarm of PSO is easy to fall into local optimal solution and has low operating efficiency. In order to conquer these defaults of PSO, many scholars have conducted various improvements and proposed some useful PSO variants [212].

2.2. Introduction of DNA Fragment. In the process of the life evolution, strengths and weaknesses of DNA will decide the life quality, so how to improve DNA quality to adapt to the environment change is a key problem for an individual evolution process. DNA is composed of some pieces where the pieces with genetic effect are called gene. In this paper, we will explore the effect of DNA + environment + interaction of environment + gene. As an individual, the ability of DNA genes to adapt to environments will be absolutely decided by its own quality and status in the existing society; thus, the micro individual can be equivalent to the microcosmic DNA.

Because of the DNA importance in individual life evolution, we regard an individual as a DNA molecule, and each dimension of an individual corresponds with DNA fragments. DNA fragments can be deemed as the internal mechanism which affects the individual behavior in society. Figure 1 gives the congruent relationship of DNA fragment with realization process of PSO.

As for the external environment, the commutation with each other and living environment of different individuals will decide its status and role in society which it belongs to, so the crossing and replacement of individual will be adopted to realize the process of the external environment effect. Figure 2 gives the effect factors of the individual evolution based on the external and internal environment change, where a swarm is composed of many single life individuals, I_Mutation and I_Crossing denote the corresponding mutation and crossover operation based on internal environment, and E_Mutation and E_Crossing are the corresponding mutation and crossover operation based on the external environment. Based on the external and internal environment change, we adopted PSO as searching engine to explore the mechanism roles.

\section{Mutation and Crossing Operation of DNA Fragments Based on Environmental Change}

3.1. Mutation Operation Based on Environment Change. In PSO, when the velocities of all particles are almost zero, the entire population will fall into local optima. The past experience shows that the mutation operation may be a good method. In [13], the author proposed that the mutation operation can produce new global best individual (gbest) to jump out of local optima. However, there are always two sides 


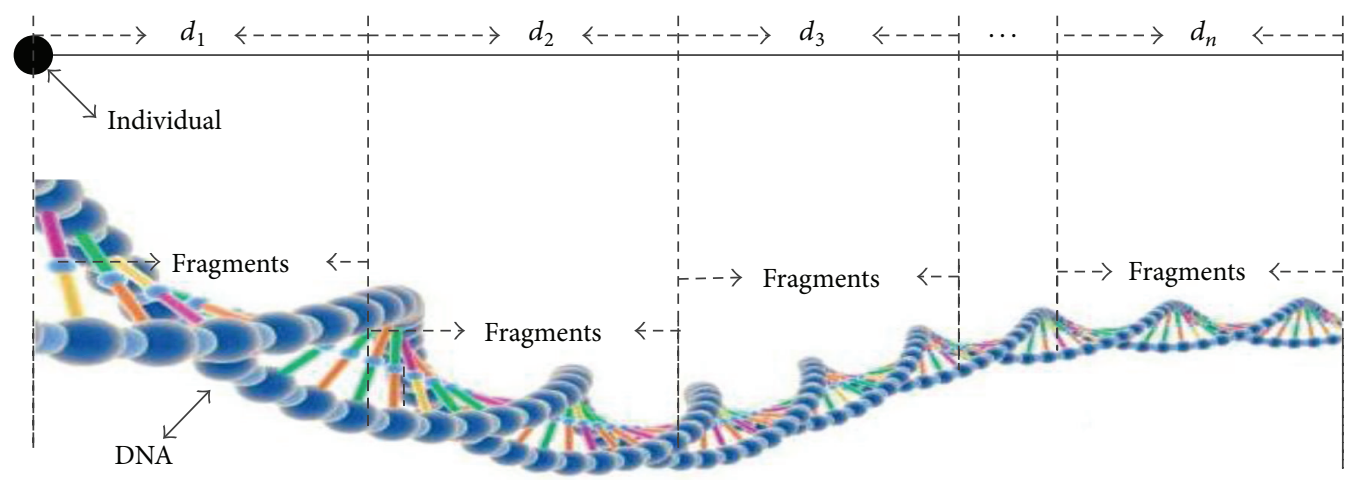

FIGURE 1: Relationship between DNA fragment and individual.

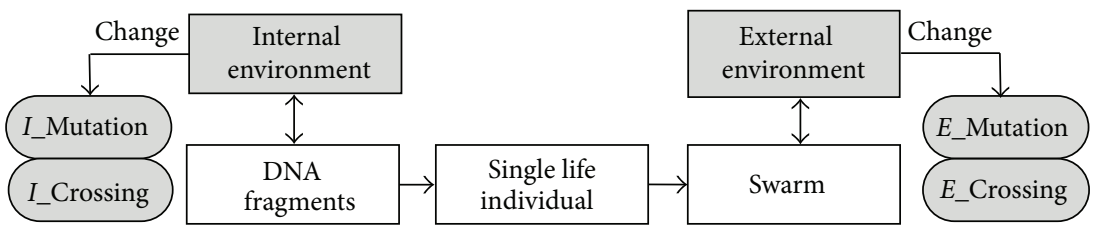

FIGURE 2: Individual evolution based on external and internal environment change.

to anything, and the new individual produced by mutilation operation may be far away from gbest. Therefore, in this paper, the mutation is divided into two types: the first one is the intermutation operation ( $I_{-}$Mutation for short) based on the internal environment change and the second one is the external mutation operation (E_Mutation for short) based on the external environment change. When the algorithm runs, the different type environment will evoke the corresponding mutation operation type.

Here, the internal and external environment change correspond to DNA fragment and swarm of PSO, respectively. As the internal environment changes, we will combine the DNA fragments and the dimension of an individual to explore their roles. Additionally, in terms of previous experiences, the phenomenon that an individual only learns from its history experience will lead to "two steps forward, one step back" [2]. Similarly, DNA fragments will have the same thing, so the internal environment change can be defined:

$$
\begin{aligned}
& D_{\text {bin }(i)}^{d}=\arg \left\{\max \left[\frac{\text { Fitness }\left(p_{j}\right)-\text { Fitness }\left(p_{i}\right)}{\left|p_{j d}-x_{i d}\right|}\right]\right\} \\
& \quad\left(i=1,2, \ldots, \text { PS; } j \in \text { neighbor }_{i}\right), \\
& D_{\text {bin }(i)}^{d} \geq \frac{\sum_{i c S} h_{i}^{d}}{N},
\end{aligned}
$$

where $\overline{D_{\operatorname{bin}(i)}}$ denotes the learning exemplar for DNA fragment of each individual; neighbor ${ }_{i}$ is the neighbor sets for individual $i$; $D_{\operatorname{bin}(i)}{ }^{d}$ is the $d$ dimension of $\overline{D_{\operatorname{bin}(i)}} ; p_{i}$ is the corresponding history best individual ( pbest $_{i}$ ); Fitness () is the solving process of the corresponding function; $\arg ()$ denotes the recognition process of DNA fragment for the corresponding individual; $h_{i}{ }^{d}$ is the $d$ dimension of individual $i ; p_{j d}$ is the $d$ dimension of individual $j$, and the other individual is similar to it; $S$ is the swarm; $N$ is the average generation count that needs to be defined. In our proposed thoughts, when $N$ is equal to or greater than a given value, the DNA fragments based on the internal environment change will be evoked. In the simulation experiments, we proposed a novel mutation strategy and the idea is the following: firstly, in the process of algorithm run, we selected some individuals (the hypothesis of the number of individuals is $N_{-} i$ ), whose pbest has no improvement for $1 / 3$ continuous total iteration (note: $1 / 3$ is the experience value); secondly, $N_{-} i$ individuals will be mutated as follows:

$$
x_{i}(t+1)=x_{i}(t)+\left(x_{i}^{U}(t)-x_{i}^{L}(t)\right) \cdot \delta_{i},
$$

where $x_{i}(t)$ with DNA fragments denotes the individual at time $t ; x_{i}{ }^{U}(t)$ and $x_{i}{ }^{L}(t)$ are upper and lower bound of $x_{i}(t)$, respectively; $\delta_{i}$ is the disturbing term of individual $i$, and its production process is as follows:

$$
\begin{aligned}
& \delta_{k}=\left(2 r_{k}\right)^{1 /\left(\eta_{m}+1\right)}-1, \quad \text { if } r_{k}<0.5, \\
& \delta_{k}=1-\left[2 \cdot\left(1-r_{k}\right)\right]^{1 /\left(\eta_{m}+1\right)}, \quad \text { if } r_{k} \geq 0.5 .
\end{aligned}
$$

Here, $r_{k}$ is the uniform random numbers in $(0,1) ; \eta_{m}$ is the mutation distribution index equal to population size.

According to the usual gene theory, the internal environment will affect the individual gene that can reflect individual performance. The external environment change will speed the gene expression, and the social group which the individual belongs to will decide the performance of the individual; for example, a person that lived in the aggressive environment will obtain the effect of the upward momentum and vice versa. Based on the thought, the external environment change can be described as follows: firstly, when 
the whole swarm has no improvement of optimization solution for $T$ generation that will be defined in terms of the problem at hand; secondly, we adopted $K$-means clustering method to select pbest of $m$ individuals (from swarm $n$ ) whose aim makes each individual have chance to develop its maximum potential; thirdly, we introduce the local search algorithm to explore some promising region in the whole search space, and here Quasi-Newton algorithm is adopted as local search engine that has the characteristics of global convergence and fast convergence. In each iteration, the search direction is determined based on Quasi-Newton algorithm as follows:

$$
\min f(x)=\frac{1}{2} x^{T} H x+b^{T}+c,
$$

where $H$ is Hessian matrix that is the positive definite symmetric matrices; $b$ is a constant vector; $c$ is a constant number. If $x^{*}$ is the optimization solution, (7) is satisfied:

$$
\begin{aligned}
\nabla f\left(x^{*}\right) & =H x+b=0, \\
x^{*} & =-H^{-1} b .
\end{aligned}
$$

The above discuss the application of the theory analysis, but in practical application of Quasi-Newton algorithm, the numerous calculations for Hessian matrix and its inverse matrix are needed. In order to effectively calculate the process, Broyden (BFGS) [4] is applied to realize the computing process whose methods are the following:

$$
\begin{aligned}
H_{k+1} & =H_{k}+\frac{q_{k} q_{k}{ }^{T}}{q_{k}{ }^{T} s_{k}}-\frac{H_{k} s_{k}{ }^{T} s_{k} H_{k}}{s_{k}{ }^{T} H_{k} s_{k}}, \\
s_{k} & =x_{k+1}-x_{k}, \\
q_{k} & =\nabla f\left(x_{k+1}\right)-\nabla f\left(x_{k}\right) .
\end{aligned}
$$

As for the computing method of Hessian matrix and its inverse matrix $H^{-1}$, DFP equation of Davidon [4] is used to solve them. As a result, each iteration can be conducted by the direction of

$$
\begin{aligned}
d & =-H_{k}^{-1} \cdot \nabla f\left(x_{k}\right), \\
x_{k+1} & =x_{k}+a_{k} d .
\end{aligned}
$$

Figure 3 gives the sketch map of local search process with Quasi-Newton algorithm and Algorithm 1 gives the whole process of Quasi-Newton algorithm.

By $K$-means clustering method, $m$ best individuals are selected as the initial search point of Quasi-Newton algorithm from the swarm $n$; then $m$ new individuals will be produced. Here, the replacement rule is the Euclidean distance; that is, $m$ new individual produced by QuasiNewton algorithm from $n$ pbest is closest to the original $m$ individual.

3.2. Crossing Operation Based on Environment Change. As a social person, his/her behavior is affected by him/herself gene and the external environment where he/she lives, so

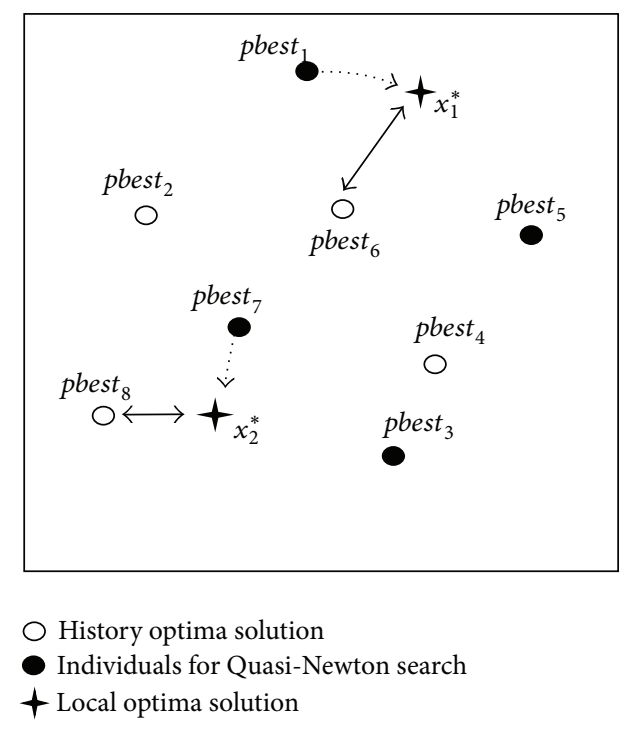

FIGURE 3: Local search process of Quasi-Newton algorithm.

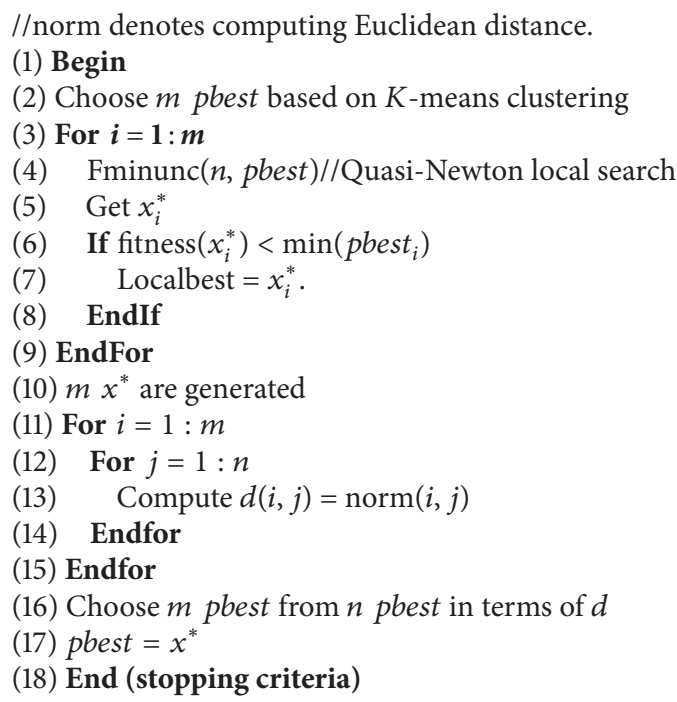

(4) Fminunc(n,pbest)//Quasi-Newton local search

(5) Get $x_{i}^{*}$

(6) If fitness $\left(x_{i}^{*}\right)<\min \left(\right.$ pbest $\left._{i}\right)$

(7) Localbest $=x_{i}^{*}$.

(8) EndIf

(9) EndFor

(10) $m x^{*}$ are generated

(11) For $i=1: m$

(12) For $j=1: n$

(13) Compute $d(i, j)=\operatorname{norm}(i, j)$

(14) Endfor

(15) Endfor

(16) Choose $m$ pbest from $n$ pbest in terms of $d$

(17) pbest $=x^{*}$

(18) End (stopping criteria)

Algorithm 1: Quasi-Newton local search.

the crossing role of his/her excellent genes and the favorable environment will improve his/her ability for better fitting society. Based on the above thoughts, we use the crossover operation to realize the process. Here, the external file is used to store the history best individual (pbest for short), and the external file can be regarded as an information store device. Note that the internal and external environment change standard is the same as the mutation standard. In the previous experiences, SBC crossing [5] is adopted to realize the crossing improvement. The internal crossing process is the following: firstly, in terms of environment change standard, the corresponding pbests are selected to conduct 
the crossover operation; secondly, the crossing operation is evoked by

$$
\begin{aligned}
& x_{1, k}=\frac{1}{2}\left[\left(1-\beta_{k}\right) \cdot x_{r, 1, k}+\left(1+\beta_{k}\right) \cdot x_{r, 2, k}\right], \\
& x_{2, k}=\frac{1}{2}\left[\left(1+\beta_{k}\right) \cdot x_{r, 1, k}+\left(1-\beta_{k}\right) \cdot x_{r, 2, k}\right],
\end{aligned}
$$

where $x_{r, i, k}$ is random individual; $x_{i, k}(i=1,2)$ is the $k$ dimension of the new individual after SBC; $\beta_{k}(\geq 0)$ is the random factor produced by (12). Here, $u$ is a random number in $(0,1) ; \eta_{c}$ is the swarm distribution index that is equal to population size. Hence,

$$
\begin{aligned}
& \beta(u)=(2 u)^{1 /\left(\eta_{c}+1\right)}, \\
& \beta(u)=[2(1-u)]^{-1 /\left(\eta_{c}+1\right)} .
\end{aligned}
$$

Thirdly, the new pbests produced by SBC operation will replace the original individuals to continue the swarm evolution process. The whole process combines the promising information of each dimension of each individual and improves the individuals to adapt to the internal environment, which is equivalent to the crossover operation of DNA fragment of the individual.

As for the crossover operation based on the external environment, its thoughts root in the real world; that is, an individual always learns from his/her history best experience and the best performance individual in his/her organization, to inherit and strengthen his/her experiences and shorten the gap with the best one. So we proposed a novel crossover operation that can produce new best history individual (pbest) by crossover operation with the global best individual (gbest). Therefore, the external crossover operation is the following: firstly, when the crossover operator is satisfied, $n$ (the swarm size) pbests and the current gbest are selected; secondly, (13) is used to cross the corresponding individual $i$ to improve their learning ability from other perfect individuals (here we called it position crossing operation). Hence,

$$
\text { pbest }_{i}=\text { gbest } i \in \text { Index. }
$$

Here, the index is defined by

$$
\begin{aligned}
& L_{i}(t)=\left\{d_{i j}(t) \mid d_{i j}(t)=\| \text { pbest }_{i}(t)-x_{j}(t) \|\right. \\
& \left.\quad+\| \text { pbest }_{i}(t)-\operatorname{gbest}(t) \|\right\}, \quad j \neq i, j \in S, \\
& N_{i}(t)=\arg \left\{\min \left[\operatorname{sort}\left(L_{i}(t)\right), n\right]\right\}, \\
& \operatorname{Index}_{i}(t)=\operatorname{find}\left\{N_{i}(t)\right\},
\end{aligned}
$$

where $L_{i}(t)$ is the sets of Euclidean distances of the current individual $i$ with other individuals and gbest at time $t$, which realized that an individual improves its own desire by learning; $S$ is the swarm size at time $t ; N_{i}(t)$ is the size of organization with the distance merit to the individual $i$ at time $t ; n$ is the number of organizations whose value is usually $1 / 5 \sim 1 / 4$ of $S$; $\operatorname{sort}(A)$ is the sort computation from small to large for sets $A$; Index ${ }_{i}$ is the finding index, which denotes the individuals nearest to individual $i$.
Finally, the $n$ new pbest will be produced by the position crossover operation, and then $n$ individual will start the new evolution.

3.3. Algorithm Design for Simulation Experiments. On the basis of the above analysis, (1) is used to update the velocity of each individual, and (15) gives the updating process of individual position, in which DNA disturbance strategies are added. We have

$$
\overline{x_{i}}(t+1)=\overline{x_{i}}(t)+\overline{v_{i}}(t+1)+\Delta D(r),
$$

where $\Delta D(r)$ is composed of $\Delta d(r)$.

In order to explore the mutation and crossing operation of DNA fragments and individual based on environmental change, the simulation experiments are conducted in single and multiobjective optimization problems. Algorithm 2 gives the pseudocode of PSO with mutation and crossing operation of DNA fragments based on environmental change in single objective optimization problem. In Algorithm 2, the data with " $\dagger$ " sign includes the required strategies of multiobjective PSO where the computing method of the external archives based on $\mathcal{\varepsilon}$-dominance and crowding distance of each particle is shown in [11].

\section{The Simulation Experiment and Analysis}

4.1. DNA Mechanism Role in Algorithm Convergence Character in Single Objection Optimization. In order to test DNA mechanism role, we adopted the combination of the qualitative and quantitative analysis for different test functions. Here, the scatter diagrams of the convergence process are used for the qualitative analysis to explore the algorithm convergence behavior, and the best optimal value and the mean value of the independent operation will be the quantitative analysis in the maximum iteration number. Additionally, we used PSO as searching engine to explore the roles of the proposed strategies, and Algorithm 2 gives the pseudocode of PSO.

For convenience, PSO with the mutation and crossover of DNA fragments and individual based on environmental change is short for DNA-PSO. Sphere, Rosenbrock, Ackley, Griewank, and Rastrigin are selected to test the algorithm performance. Maximum number of iterations of all algorithms (MAXT) is 1000. Population size (PS) is 30. Function dimension is 60 . In order to test the DNA mechanism role in the algorithm stability in single objection optimization problem, we will rotate Ackley, Griewank, Rastrigin, and Rastrigin_noncont function to further test the effectiveness of the proposed strategies, and the fitness evaluation of the rotated function is $6 \times 10^{4}$. In simulation experiments, we will compare the performances of DNA-PSO with CF-LPSO [1], CF-GPSO [1], and CPSO [2]. Each algorithm is implemented in Matlab R2008B platform. Figures 4 and 5 show the algorithm convergence characteristics in the nonrotated and rotated function.

On nonrotated function, except for Sphere function, DNA-PSO achieved the best performance, especially on multimodal function; DNA mechanism has obvious merit. 
(1) Begin

(2) Initialize positions and associated velocity of all particles

(3) Evaluate the fitness values of all particles

(4) Set the current position as pbest

(5) Set $V_{\max }=0.25\left(X_{\max }-X_{\min }\right)$

$(6)^{\dagger}$ Build initial external archives based on $\varepsilon$-dominance

$(7)^{\dagger} \quad$ Calculate crowding distance of each particle

(8) While (fitcout $<$ Max_FES) $\& \&$ ( $\boldsymbol{k}<$ iteration)

(9) For each particle ( $i=1$ : PS)

(10) Update particle velocity and position by (1) and (15)

(11) Update pbest

(12) Evaluate the fitness values of current particle $i$

(13) End for

(14) If internal environment change

(15) Run $I \_$Mutation and I_Crossing

(16) End if

(17) If external environment change

(18) Run E_Mutation and E_Crossing

(19) End if

(20) Updating the relative parameters

(21) Increase the generation count

$(22)^{\dagger} \quad$ Update the external archive

(23) End While

(24) Output the results

(25) End Begin

Algorithm 2

TABLE 1: Means and confidence interval for nonrotating function after $3 \times 10^{4}$ function evaluations.

\begin{tabular}{lccccc}
\hline Algorithm & Sphere & Rosenbrock & Ackley & Griewank & Rastrigin \\
\hline \multirow{2}{*}{ CF-LPSO } & $4.5502 e-017 \pm$ & $2.4218 e+001 \pm$ & $1.8978 e+000 \pm$ & $4.6610 e-002 \pm$ & $5.1738 e+001 \pm$ \\
& $1.3201 e-017$ & $1.1236 e+001$ & $1.1374 e+000$ & $3.7631 e-002$ & $2.5426 e+001$ \\
\hline \multirow{2}{*}{ CF-GPSO } & $4.7546 e-014 \pm$ & $2.4078 e+001 \pm$ & $1.3769 e+001 \pm$ & $3.2886 e-001 \pm$ & $8.2581 e+001 \pm$ \\
& $2.3421 e-014$ & $1.7456 e+001$ & $2.8168 e+001$ & $1.4561 e-001$ & $1.6346 e+001$ \\
\hline \multirow{2}{*}{ CPSO } & $3.0583 e-006 \pm$ & $2.4899 e+000 \pm$ & $2.5444 e-004 \pm$ & $2.2157 e-002 \pm$ & $1.9938 e+000 \pm$ \\
& $3.2143 e-006$ & $1.1361 e+000$ & $1.2432 e-004$ & $1.5632 e-002$ & $1.7831 e+000$ \\
\hline \multirow{2}{*}{ DNA-PSO } & $7.9834 e-017 \pm$ & $7.3967 e-001 \pm$ & $6.3452 e-005 \pm$ & $1.0015 e-006 \pm$ & $1.1902 e-004 \pm$ \\
& $3.2902 e-017$ & $0.8932 e-001$ & $1.0098 e-005$ & $0.8932 e-006$ & $0.2341 e-004$ \\
\hline Result & 0 & 1 & 1 & 1 & 1 \\
\hline
\end{tabular}

Similarly, in rotated function (Figure 5), DNA-PSO has also strong competitiveness.

Table 1 gives the mean and 95\% confidence interval after $3 \times 10^{4}$ function evaluations for nonrotated functions, and Table 2 gives the mean and 95\% confidence interval after $6 \times$ $10^{4}$ function evaluations for rotated functions. In order to test the difference of the results of different algorithms from the statistical significance, we adopted Wilcoxon rank to analyze the results of DNA-PSO with the other PSOs in the optimal test results, and the statistical test results appear at the bottom of Tables 1 and 2.

From Figures 4 and 5, we can clearly see that DNA-PSO has better convergence characteristic compared with PSOs, and particularly in the rotated function, DNA-PSO shows better ability to jump out of local optima. Better performance of DNA-PSO roots in the mutation and crossover operation of DNA fragments based on the environmental change, where the mechanism of mutation and crossover can take full advantage of each DNA fragment of an individual, to effectively avoid "two steps forward, one step back" phenomenon. The mutation and crossover operator for the external environmental change will make pbest of each individual and gbest of the whole swarm be of sufficient use and blend. Additionally, from the quantitative analysis (Tables 1 and 2) and statistical analysis, we can also get the same conclusion. Therefore, whether qualitative or quantitative analysis, the mutation and crossover operation of DNA fragments and each individual based on the environmental change can improve the swarm intelligence performance, and as a result, the strategies can be an effective improvement method for the algorithm design and variants.

4.2. DNA Mechanism Role in Algorithm Convergence Character in Multiobjection Optimization. In the single objective 


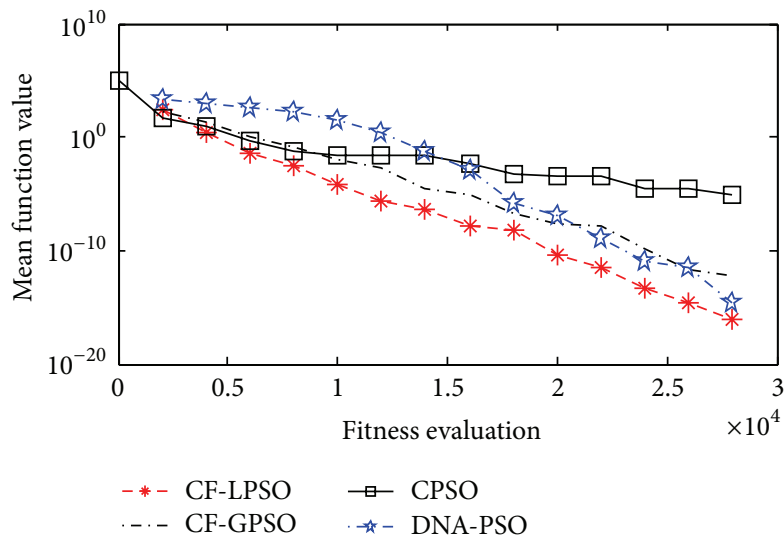

(a) Sphere function

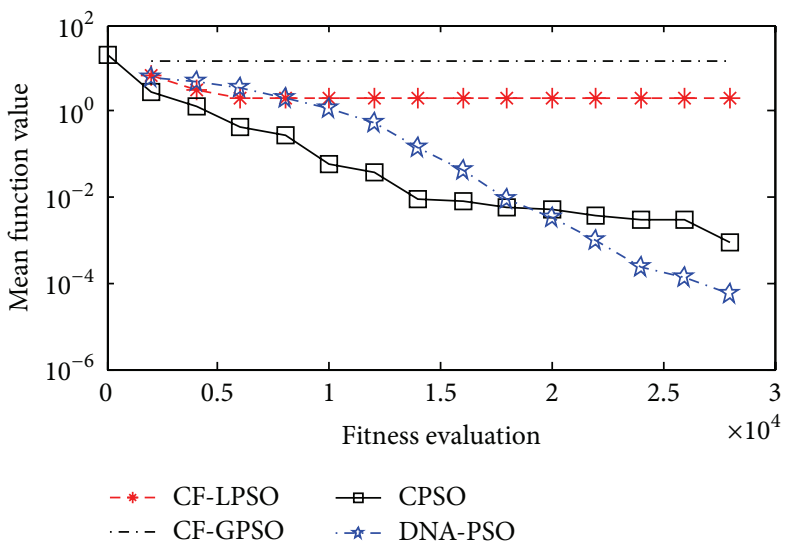

(c) Ackley function

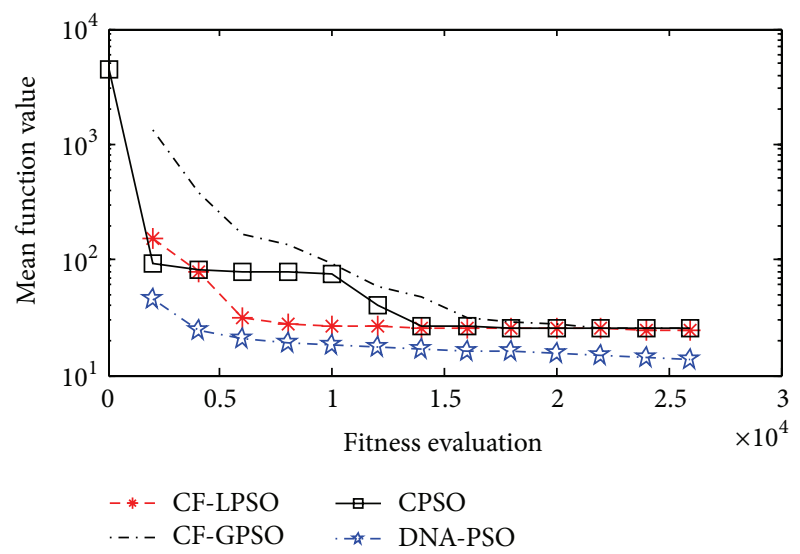

(b) Rosenbrock function

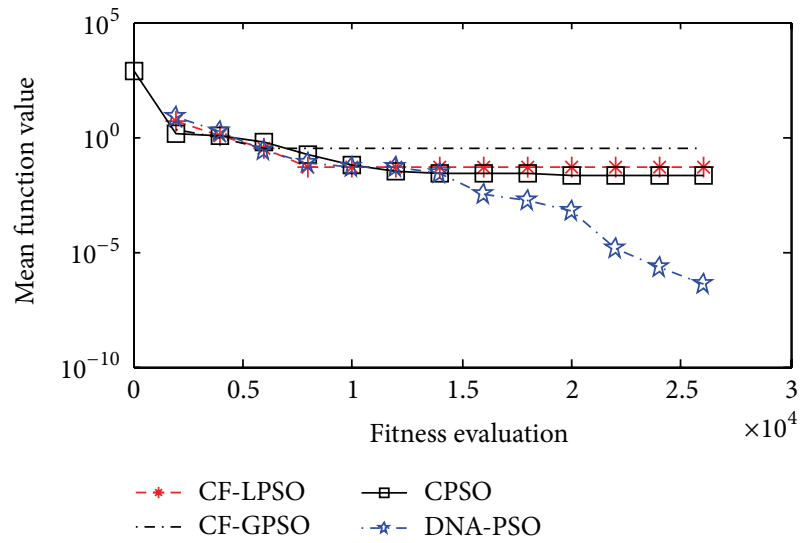

(d) Griewank function

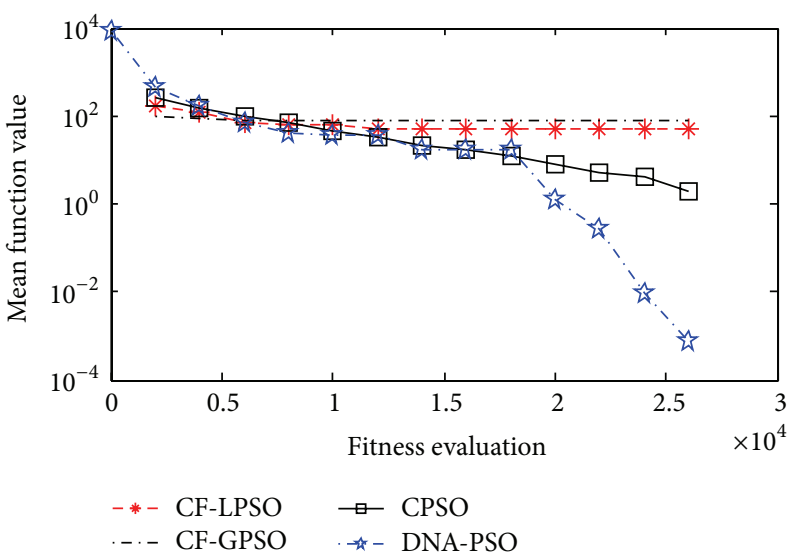

(e) Rastrigin function

FIGURE 4: Nonrotated function convergence figure.

optimization problem, the mechanisms of the mutation and crossover operation of DNA fragments based on the internal environmental change have shown positive role; to further test their performance, we also choose two representative multiobjective PSOs (MOPSO [11] and OMOPSO [12]) and four test functions (ZDT1, ZDT2, ZDT3, and ZDT4) to test the proposed mechanism role in multiobjective swarm intelligence algorithm. In this section, the dimension of decision variables of each test function is 30. Figures 6-9 clearly show that DNA-PSO has the ability to trace Pareto fronts which implies that DNA-PSO is effective for solving multiobjection problem. Particularly, as for ZDT4 function with $21^{9}$ local optima, DNA-PSO has better performance to find and trace its Pareto front compared to MOPSO and OMOPSO.

\section{Conclusions and Future Work}

This paper mainly discussed the strategies of the mutation and crossover operation of DNA fragments and individuals 


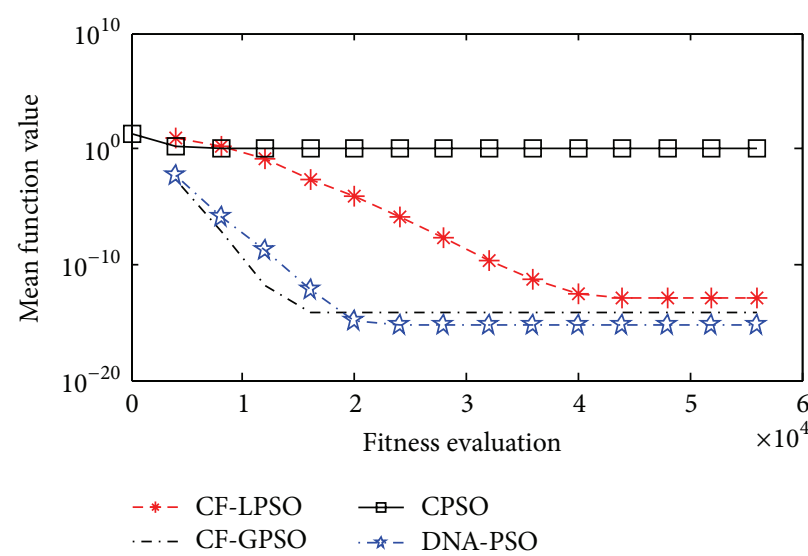

(a) Ackley function

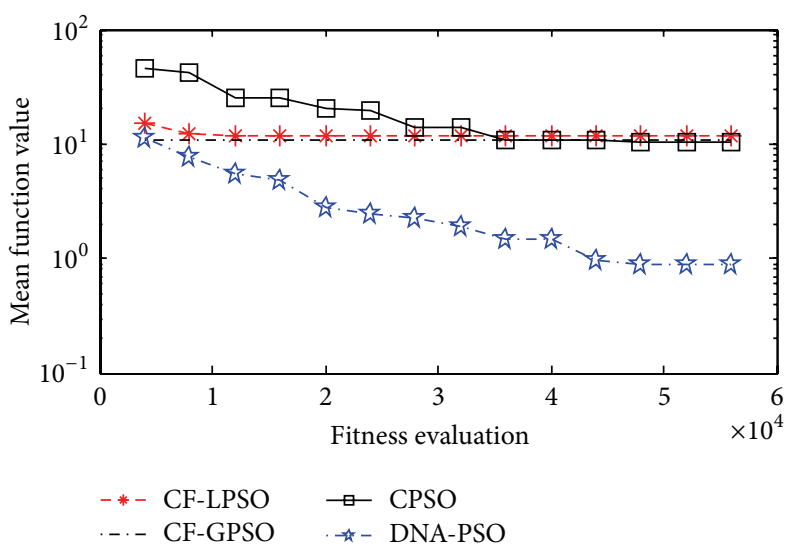

(c) Rastrigin function

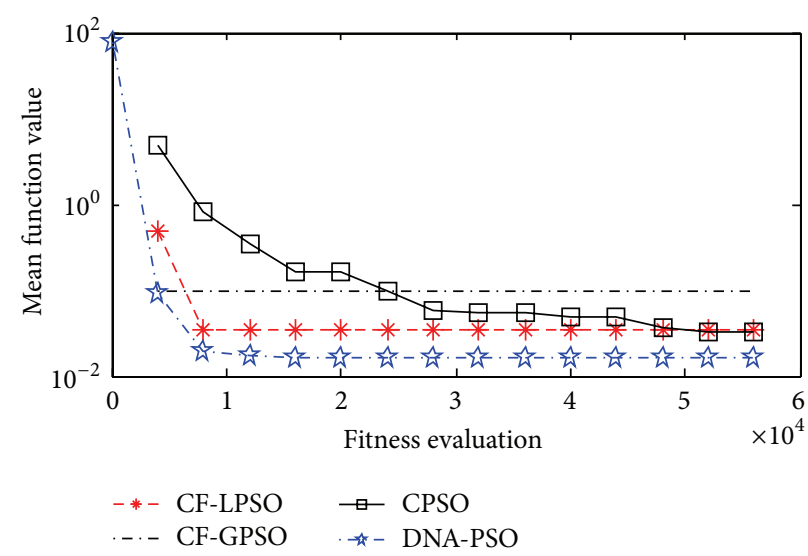

(b) Griewank function

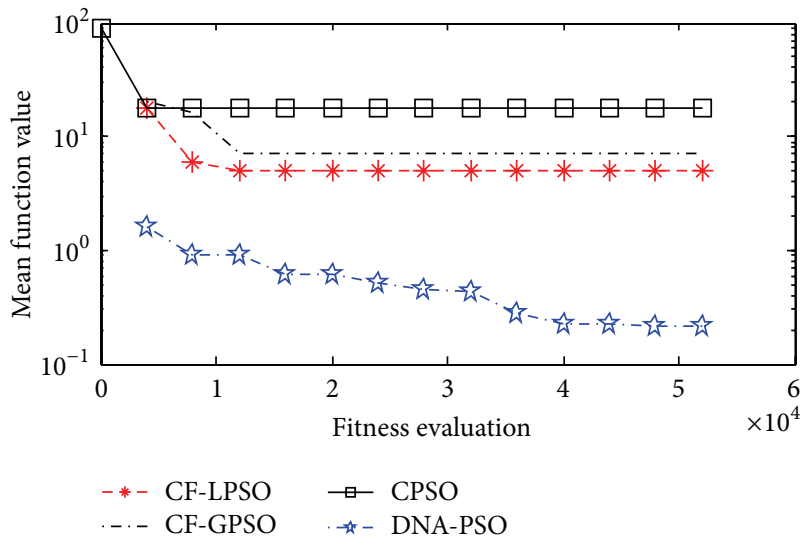

(d) Rastrigin_noncont function

FIGURE 5: Rotated function convergence figure.

TABLE 2: Means and confidence interval for rotating function after $6 \times 10^{4}$ function evaluations.

\begin{tabular}{lcccc}
\hline Algorithm & Ackley & Griewank & Rastrigin & Rastrigin_noncont \\
\hline \multirow{2}{*}{ CF-LPSO } & $1.3856 e-013 \pm$ & $3.4467 e-002 \pm$ & $1.1939 e+001 \pm$ & $5.3000 e+000 \pm$ \\
& $1.5367 e-013$ & $1.4325 e-002$ & $1.5453 e+001$ & $1.2454 e+000$ \\
\hline \multirow{2}{*}{ CF-GPSO } & $7.1054 e-015 \pm$ & $9.8354 e-002 \pm$ & $1.0945 e+001 \pm$ & $1.4341 e+001$ \\
& $3.5346 e-015$ & $2.6572 e-002$ & $1.0228 e+001 \pm$ & $2.4325 e+000$ \\
\hline \multirow{2}{*}{ CPSO } & $1.1551 e+000 \pm$ & $3.1611 e-002 \pm$ & $1.5412 e+001$ & $1.800 e+001 \pm$ \\
& $1.6366 e+000$ & $5.5432 e-002$ & $6.6683 e-001 \pm$ & $3.0565 e+001$ \\
\hline \multirow{2}{*}{ DNA-PSO } & $4.0982 e-016 \pm$ & $1.2098 e-002 \pm$ & $5.0192 e-001 \pm$ \\
& $2.0097 e-016$ & $1.0021 e-002$ & $2.3894 e-001$ & $1.1102 e-001$ \\
\hline Result & 0 & 1 & 0 & 1
\end{tabular}

based on the environmental change, and these strategies are embedded to particle swarm optimization (DNA-PSO for short). In order to test the effectiveness of the proposed strategies, we design DNA-PSO that can deal with single and multiobjective optimization algorithm by adding some methods and conducted the simulation experiments in single objective problems (Sphere, Rosenbrock, Ackley, Griewank, and Rastrigin) and multiobjective test problems (ZDT1, ZDT2, ZDT3, and ZDT4). Additionally, in order to test DNA-PSO stability in single objection optimization, we rotated Ackley, Griewank, Rastrigin, and Rastrigin_noncont function. Simulation experiments show that, whether qualitative or quantitative analysis, the mutation and crossover operation of DNA fragments and individuals based on environmental change can improve the swarm intelligence performance, and they are effective methods for algorithm design and variants. Finally, in view of the effectiveness of the proposed strategies, in the future, we will focus 


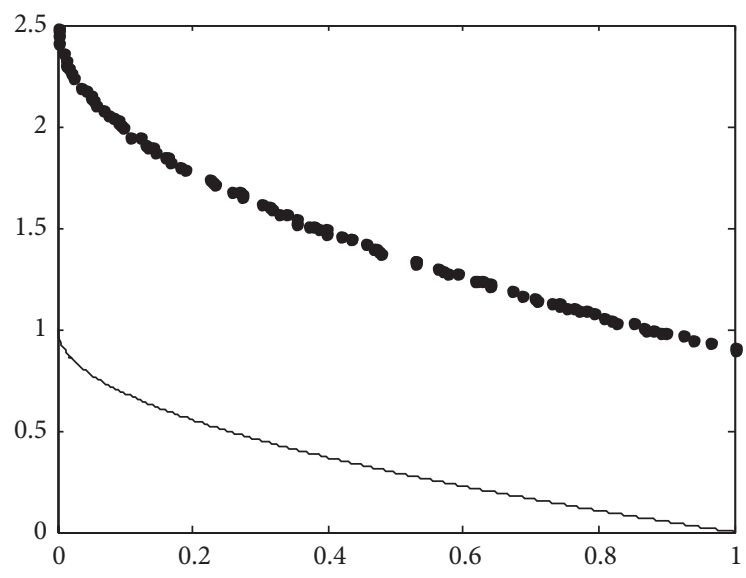

- True Pareto front

- MOPSO

(a)

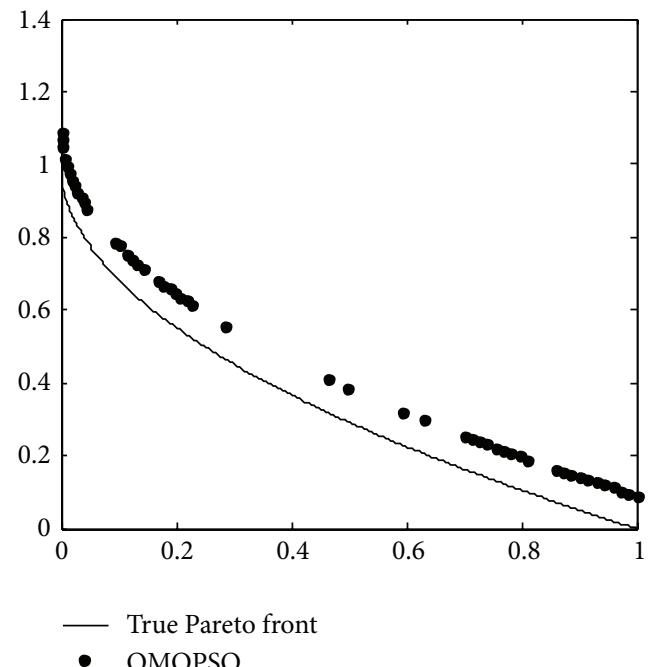

(b)

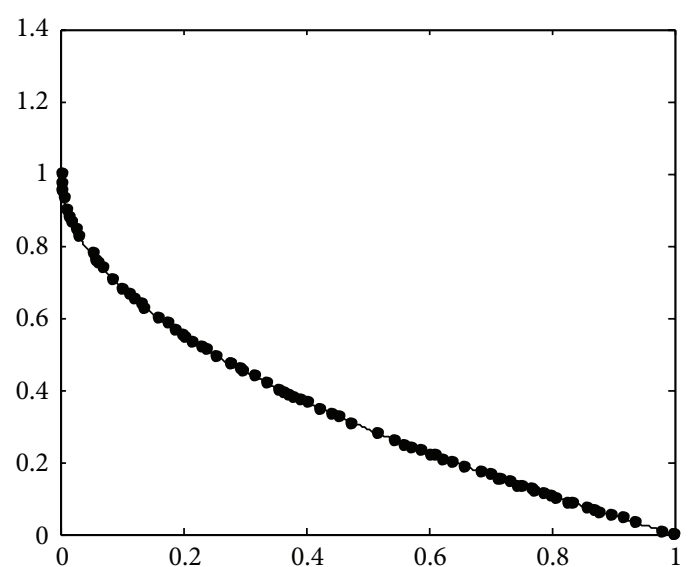

True Pareto front

- DNA-PSO

(c)

FIgURE 6: Pareto fronts obtained in test problem ZDT1.

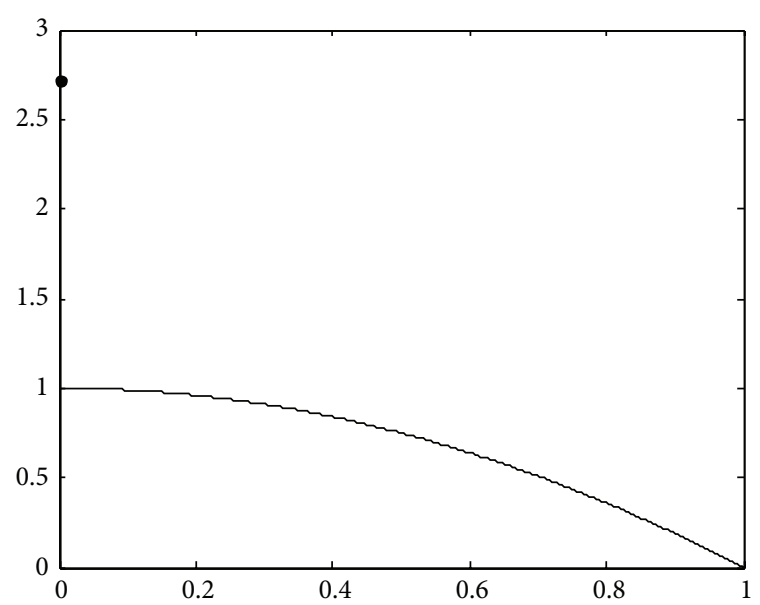

- True Pareto front

- MOPSO

(a)

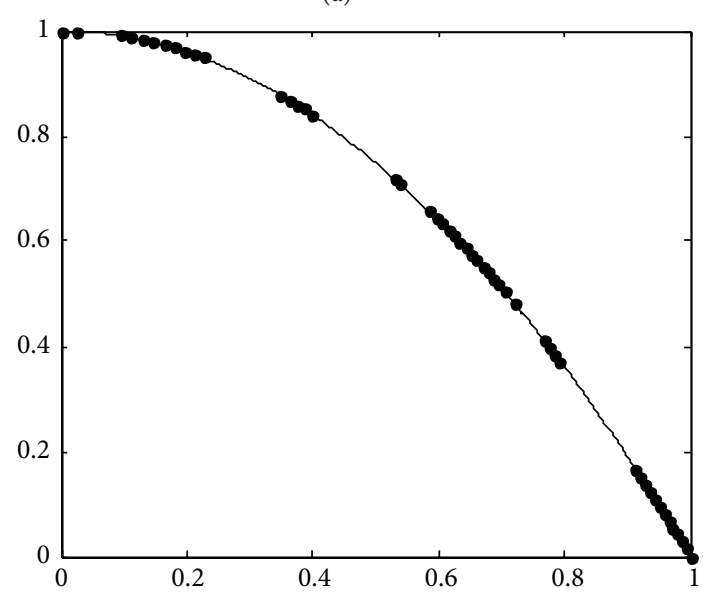

- True Pareto front

- OMOPSO

(b)

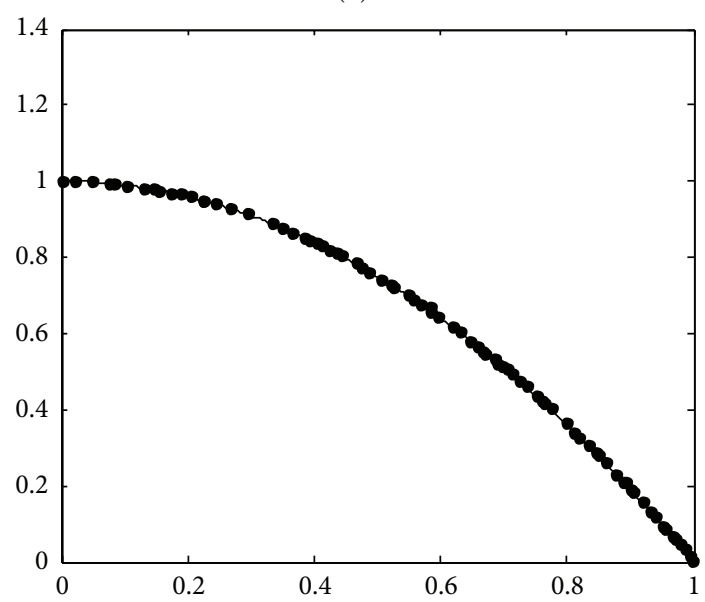

True Pareto front

- DNA-PSO

(c)

FIgURE 7: Pareto fronts obtained in test problem ZDT2. 


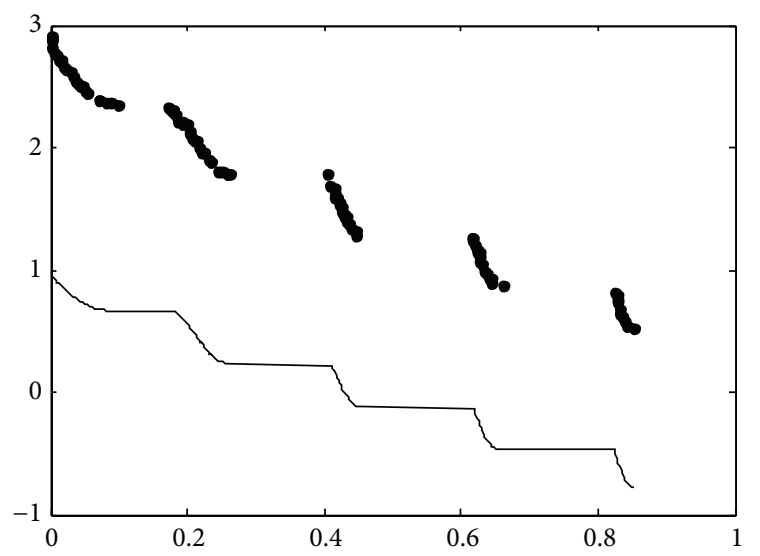

_ True Pareto front

- MOPSO

(a)

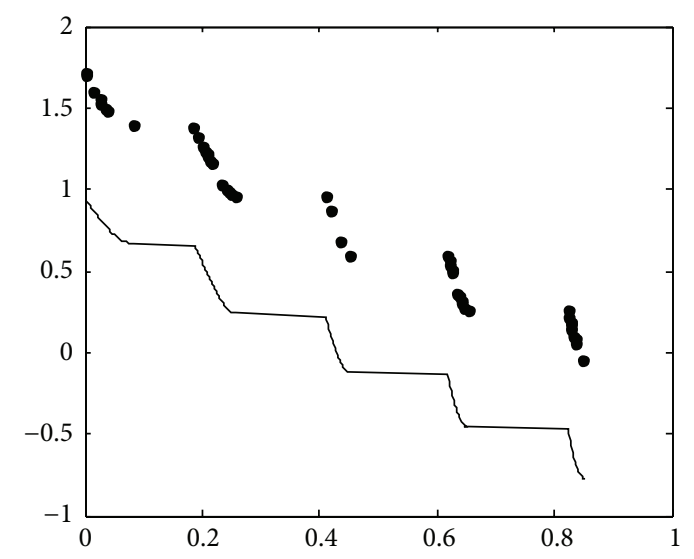

— True Pareto front

- OMOPSO

(b)

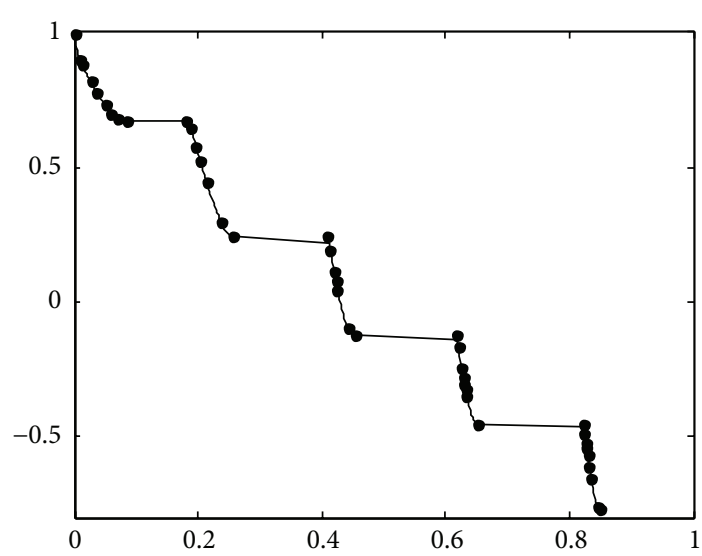

- True Pareto front

- DNA-PSO

(c)

FIGURE 8: Pareto fronts obtained in test problem ZDT3.

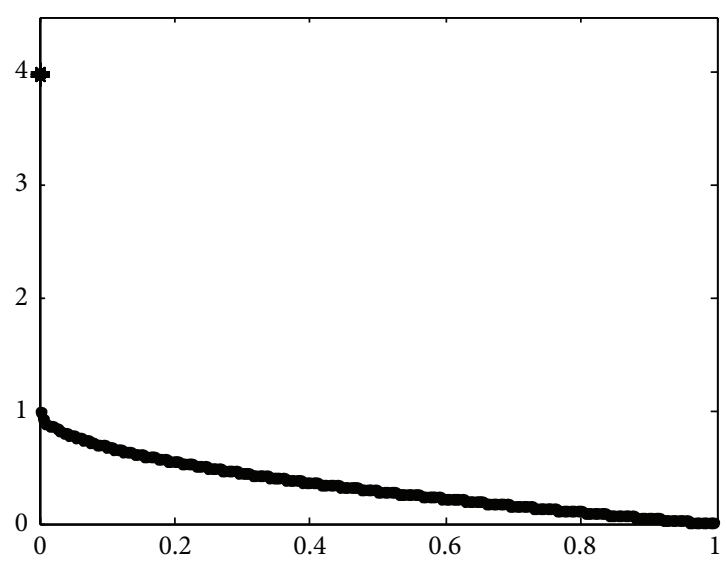

- True Pareto front

* MOPSO

(a)

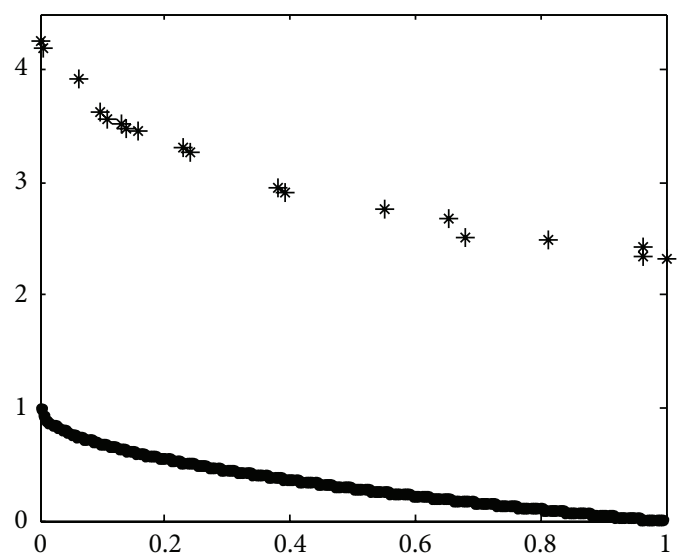

- True Pareto front

* OMOPSO

(b)

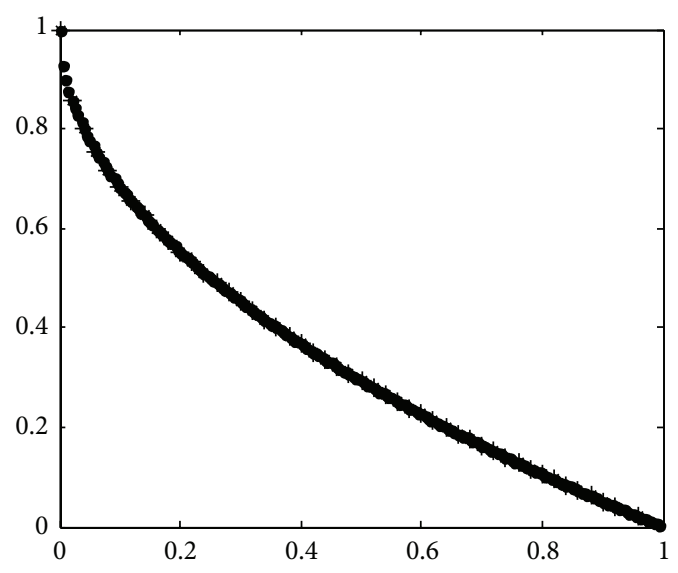

- True Pareto front

* DNA-PSO

(c)

FIgURE 9: Pareto fronts obtained in test problem ZDT4. 
on (i) proposing a novel intelligence algorithm based on DNA mechanism and (ii) exploring new biology role in life evolution.

\section{Conflict of Interests}

The authors declare that there is no conflict of interests regarding the publication of this paper.

\section{Acknowledgments}

This work is supported by the National Natural Science Foundation of China (Grants nos. 71461027, 71471158, 71001072, and 71271140); Guizhou Province Science and Technology Fund (Qian Ke He J [2012] 2340 and [2012] 2342, LKZS [2012] 10 and [2012] 22, [2015] 7005, [2014] 29); Guizhou Province Natural Science Foundation in China (Qian Jiao He KY [2014] 295, Qian Jiao He Zhong da Zhuang Xiang $\mathrm{Zi}$ [2014] 036); the Educational Reform Project in Guizhou Province Department of Education (Qian Jiao Gao Fa [2013] 446); Guizhou Province College Students' Innovative Entrepreneurial Training Plan (201410664004); 2013 and 2014 Zunyi 15851 Talents Elite Project Funding; Science and Technology Talent Training Object of Guizhou Province Outstanding Youth (Qian Ke He Ren Zi [2015] 06).

\section{References}

[1] J. Kennedy and R. Eberhart, "Particle swarm optimization," in Proceedings of the IEEE International Conference on Neural Networks, vol. 4, pp. 1942-1948, December 1995.

[2] F. Van Den Bergh and A. P. Engelbrecht, "A Cooperative approach to particle swarm optimization," IEEE Transactions on Evolutionary Computation, vol. 8, no. 3, pp. 225-239, 2004.

[3] S. Z. Martínez and C. A. Coello Coello, "Hybridizing an evolutionary algorithm with mathematical programming techniques for multi-objective optimization," in Proceedings of the 10th Annual Genetic and Evolutionary Computation Conference (GECCO '08), pp. 769-770, New York, NY, USA, July 2008.

[4] C. G. Broyden, "The convergence of a class of double-rank minimization algorithms," Journal of the Institute of Mathematics and Applications, vol. 6, no. 7, pp. 76-90, 1970.

[5] K. Deb and R. B. Agrawal, "Simulated binary crossover for continuous search space," Complex Systems, vol. 9, no. 2, pp. 115148, 1995.

[6] F. van den Bergh and A. P. Engelbrecht, "A cooperative approach to participle swam optimization," IEEE Transactions on Evolutionary Computation, vol. 8, no. 3, pp. 225-239, 2004.

[7] B. Niu and L. Li, "A novel PSO-DE-based hybrid algorithm for global optimization," in Advanced Intelligent Computing Theories and Applications. With Aspects of Artificial Intelligence: 4th International Conference on Intelligent Computing, ICIC 2008 Shanghai, China, September 15-18, 2008 Proceedings, vol. 5227 of Lecture Notes in Artificial Intelligence, pp. 156-163, Springer, Berlin, Germany, 2008.

[8] J. J. Liang, A. K. Qin, P. N. Suganthan, and S. Baskar, "Comprehensive learning particle swarm optimizer for global optimization of multimodal functions," IEEE Transactions on Evolutionary Computation, vol. 10, no. 3, pp. 281-295, 2006.
[9] T. Peram and K. Veeramachaneni, "Fitness-distance-ratio based particle swarm optimization," in Proceedings of the IEEE Swarm Intelligence Symposium, pp. 174-181, IEEE, Indianapolis, Ind, USA, April 2003.

[10] M. Clerc and J. Kennedy, "The particle swarm-explosion, stability, and convergence in a multidimensional complex space," IEEE Transactions on Evolutionary Computation, vol. 6, no. 1, pp. 58-73, 2002.

[11] C. A. C. Coello, G. T. Pulido, and M. S. Lechuga, "Handling multiple objectives with particle swarm optimization," IEEE Transactions on Evolutionary Computation, vol. 8, no. 3, pp. 256-279, 2004.

[12] M. R. Sierra and C. A. C. Coello, "Improving PSO-based multi-objective optimization using crowding, mutation and $\varepsilon$-dominance," in Evolutionary Multi-Criterion Optimization: Third International Conference, EMO 2005, Guanajuato, Mexico, March 9-11, 2005. Proceedings, vol. 3410 of Lecture Notes in Computer Science, pp. 505-519, Springer, 2005.

[13] A. Stacey, M. Jancic, and I. Grundy, "Particle swarm optimization with mutation," in Proceedings of the Congress on Evolutionary Computation (CEC '03), vol. 2, pp. 1425-1430, IEEE, Canberra, Australia, December 2003. 


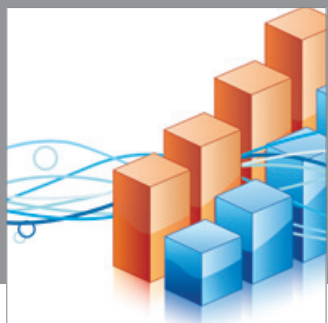

Advances in

Operations Research

mansans

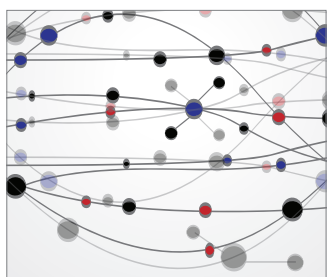

The Scientific World Journal
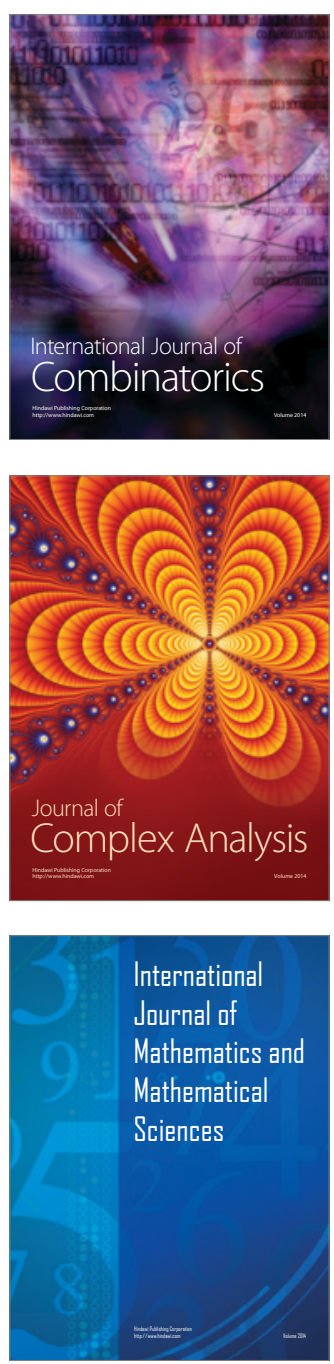
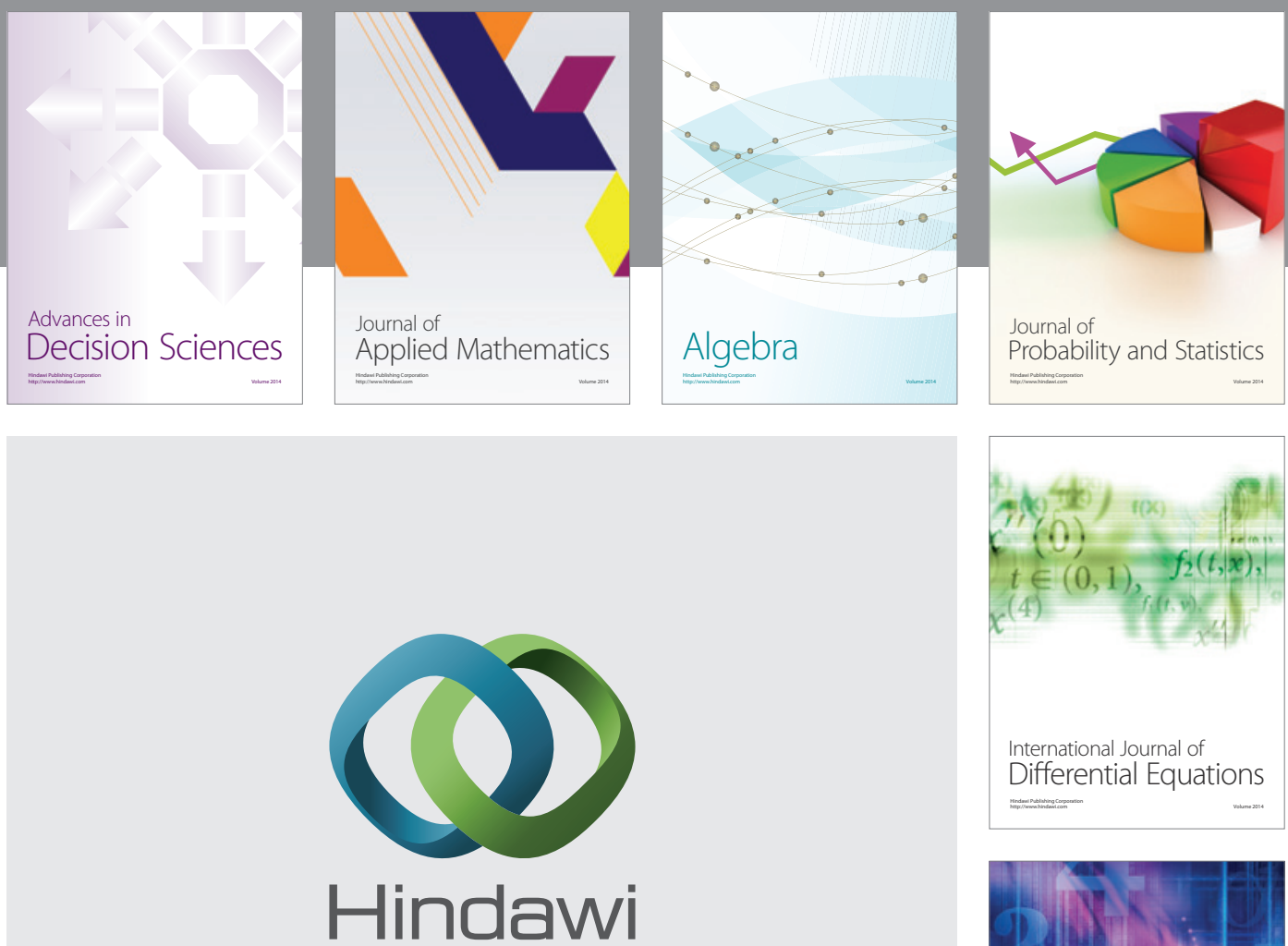

Submit your manuscripts at http://www.hindawi.com
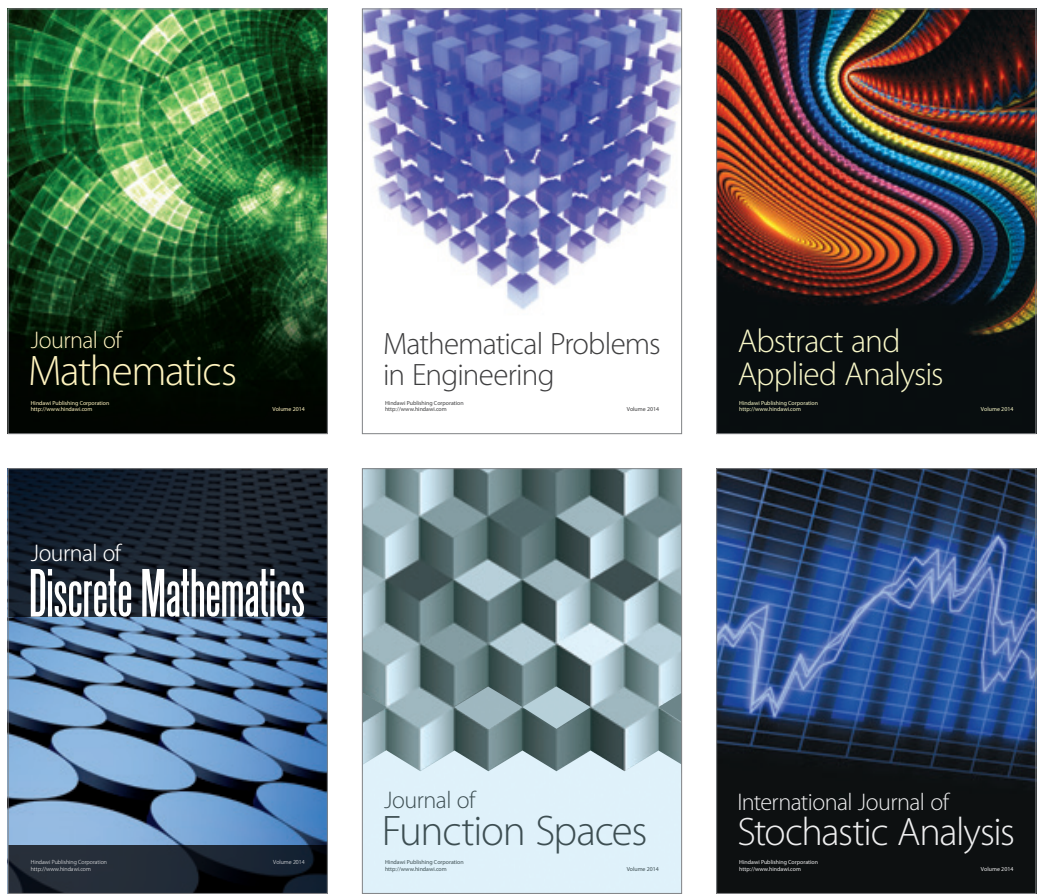

Journal of

Function Spaces

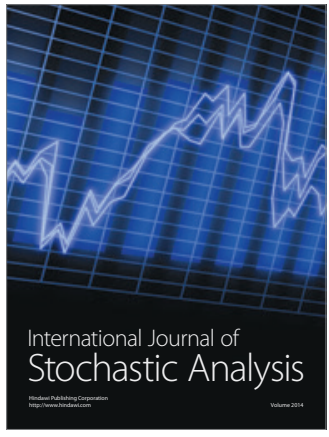

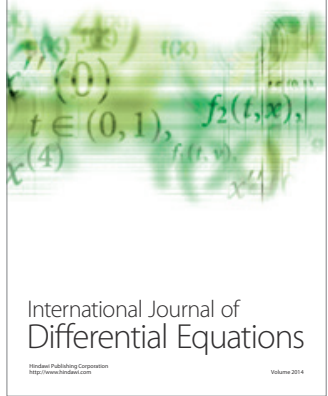
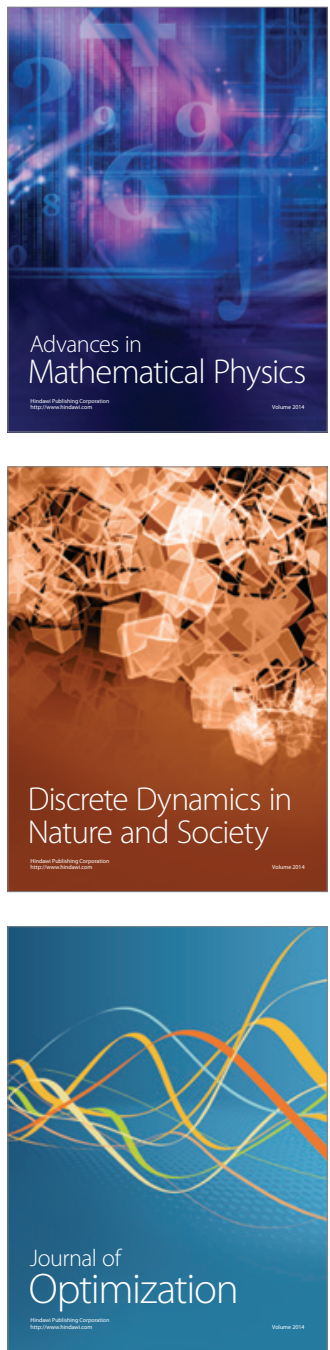